Luís Henrique Garcia Muniz'

Robson Marcelo Rossi"

Herintha Coeto Neitzke"I

Wuelton Marcelo Monteiro'"'

Ueslei Teodoro'

\section{Estudo dos hábitos alimentares de flebotomíneos em área rural no sul do Brasil}

\section{H ost feeding preferences of sandflies in rural area, Southern Brazil}

OBJETIVO: Investigar a composição específica e aspectos da preferência alimentar de flebotomíneos em relação aos animais domésticos existentes em área endêmica de leishmaniose tegumentar americana.

MÉTODOS: Os flebotomíneos foram coletados nos meses de janeiro a abril de 2004, das 20 às 24h, numa área situada a $40 \mathrm{~m}$ de uma das residências de um sítio localizado no município de Mandaguari, PR, sul do Brasil. Foram usadas quatro armadilhas luminosas de Falcão, instaladas a $5 \mathrm{~m}$ de distância uma da outra, ao lado de uma gaiola, cada uma delas contendo isca animal (suíno, cão, coelho e galinha).

RESULTADOS: Foram coletados 1.697 exemplares de flebotomíneos, das espécies: Nyssomyia whitmani, Pintomyia fischeri, Migonemyia migonei, Nyssomyia neivai, Pintomyia pessoai e Psathromyia shannoni, predominando N. whitmani. Não houve preferência alimentar dos flebotomíneos em relação aos animais investigados.

CONCLUSÕES: Verificou-se que $N$. whitmani e $P$. fischeri são oportunistas e, provavelmente, as fêmeas ajustam os seus hábitos alimentares à disponibilidade de hospedeiros, sugerindo o ecletismo alimentar desstes insetos nos ambientes antrópicos.

DESCRITO RES: Preferências alimentares. Psychodidae. Insetos vetores. Leishmaniose tegumentar americana, epidemiologia. Leishmaniose cutânea. Flebotomíneos. N. whitmani. P. fischeri.

\title{
ABSTRACT
}

Programa de Pós-Graduação em Análises Clínicas. Universidade Estadual de Maringá (UEM). Maringá, PR, Brasil

"Departamento de Estatística. UEM. Maringá, PR, Brasil

III Curso de Farmácia. UEM. Maringá, PR, Brasil

Correspondência | Correspondence:

Luís Henrique Garcia Muniz

R. Renê Tácola, 708 apto 1102

86975-000 Mandaguari, PR, Brasil

E-mail: Ingmuniz@turbopro.com.br

Recebido: 3/3/2005 Revisado: 4/7/2006 Aprovado: 31/8/2006
OBJECTIVE: To investigate the fauna and host feeding preferences of sandflies as for domestic animals in an endemic area of American cutaneous leishmaniasis.

METHODS: The sandflies collections were carried out from 8:00 pm to 12:00 am in an area 40 meters away from one of the residences in a farm in Southern Brazil, between January and April, 2004. Collection was performed using four Falcão light traps, placed $5 \mathrm{~m}$ apart from each other beside a cage, each one of them containing an animal bait (swine, dog, rabbit, and chicken).

RESULTS: A total of 1,697 specimens of sandflies were collected as follows: Nyssomyia whitmani, Pintomyia fischeri, Migonemyia migonei, Nyssomyia neivai, Pintomyia pessoai and Psathromyia shannoni. The prevalent species was $N$. whitmani. There were no feeding preferences of sandflies as for the animals studied.

CONCLUSIONS: $N$. whitmani and P. fischeri are opportunistic species and female 
insects probably adjust their eating habits to host availability, suggesting eating eclecticism of these insects in anthropic environments.

\section{KEYW ORDS: Food preferences. Psychodidae. Insect vectors. American cutaneous leishmaniasis, epidemiology. Leishmaniasis, cutaneous. Sandflies. N. whitmani. P. fischeri.}

\section{INTRO DUÇÃO}

A leishmaniose tegumentar americana (LTA) é uma doença endêmica em vários Estados do Brasil, com 552.059 casos humanos notificados entre 1980 e 2003.* Nesse mesmo período, foram notificados 12.220 casos de LTA no Estado do Paraná,* distribuídos em diversos municípios, ${ }^{8}$ representando 99,3\% dos 12.304 casos registrados na região Sul do País e 2,2\% dos casos registrados no Brasil.

As ações humanas sobre o meio ambiente atuam na seleção das espécies de flebotomíneos e mamíferos reservatórios de Leishmania, permitindo àqueles com maior valência ecológica se adaptarem ao ambiente antrópico. Essas ações parecem favorecer a presença desses insetos e mamíferos no domicílio e peridomicílio, explicando, em parte, a persistência das leishmanioses nesse tipo de ambiente. Além disso, as habitações humanas de má qualidade e em locais inadequados, a construção desordenada de abrigos de animais domésticos no ambiente peridomiciliar e a carência de condições mínimas de saneamento básico são condições comuns em áreas rurais e periféricas de centros urbanos. ${ }^{8,12-14}$ Nessas áreas, os mamíferos reservatórios de Leishmania têm sobrevivido e os flebotomíneos têm sido capturados em grande número, ${ }^{12-15}$ favorecendo a infecção humana e de animais domésticos.

No norte do Estado do Paraná, onde as alterações ambientais vêm ocorrendo de maneira acentuada desde a década de 40, a LTA é endêmica e apresenta fortes indícios de que a infecção humana está ocorrendo no ambiente extraflorestal, no domicílio e peridomícilio. ${ }^{8,12}$ De modo geral, nas localidades rurais desse Estado, onde os casos de LTA têm surgido com freqüência, o espaço humano ocupado pelo domicílio e peridomicílio guarda semelhança com aqueles ambientes propícios à transmissão de leishmanioses.

O hábito alimentar de flebotomíneos tem sido estu- dado por diversos pesquisadores, os quais observaram que mesmo havendo afinidade dos flebotomíneos por determinadas fontes alimentares, esses dípteros mostravam-se oportunistas, uma vez que sugavam sangue de diversos mamíferos.**,1,3,5,11,16,17 Os estudos das fontes sangüíneas de flebotomíneos têm sido muito úteis no esclarecimento da epidemiologia das leishmanioses, podendo vir a auxiliar as atividades de controle e vigilância dessas doenças. ${ }^{6}$

No município de Mandaguari ocorreram quatro casos autóctones de LTA em 2003 e quatro em 2004. No Sítio Flor de Maio, com um ambiente favorável à transmissão de Leishmania, uma moradora foi infectada no primeiro semestre de 2003. Este fato levou à realização do presente estudo, com o objetivo de investigar a fauna e os aspectos da preferência alimentar de flebotomíneos em relação aos animais domésticos.

\section{MÉTODOS}

A pesquisa foi realizada no sítio Flor de Maio, município de Mandaguari, localizado a 23⒊ $31^{\prime}$ S e 51²41'W, no norte do Paraná, sul do Brasil. O clima é do tipo subtropical úmido mesotérmico, com verões quentes e tendência de concentração de chuvas (temperatura média superior a $22^{\circ} \mathrm{C}$ ); inverno com geadas pouco freqüentes (temperatura média inferior a $18^{\circ} \mathrm{C}$ ), sem períodos de seca definidos. A precipitação anual variou de 1.853 mm em 1997 a 1.613 mm em 2004, com média anual de $1.742,4 \mathrm{~mm}$. Nesse período, o mês mais chuvoso foi fevereiro com a média de $222,9 \mathrm{~mm}$ e os meses mais secos, julho e agosto, com médias de $61,8 \mathrm{~mm}$ e $59,1 \mathrm{~mm}$, respectivamente. $* * *$

Com o desenvolvimento da agricultura, a mata do tipo Floresta Estacional Semidecidual foi quase completamente devastada. Hoje a ocupação das terras do município compreende lavouras de soja, café, milho, trigo, cana-de-açúcar, pastagens e outras.

*Ministério da Saúde, Secretaria de Vigilância em Saúde. Série histórica de casos de doenças de notificação compulsória por doença (19802005). Disponível em http://portal.saude.gov.br/portal/arquivos/pdf/planilhas dnc casos e obitos todas 2006.pdf [acesso em 17 out 2006] **Casanova C. Avaliação do potencial biológico de Lutzomyia intermedia s. I. cōmo vētor de leishmaniose tegumentar americana na zona rural do município de Conchal, estado de São Paulo [tese de doutorado]. São Paulo: Faculdade de Saúde Pública da USP; 2001. ***Informações fornecidas pela Cooperativa dos Cafeicultores de Mandaguari (COCARI). 
As capturas de flebotomíneos foram realizadas a aproximadamente $1,5 \mathrm{~km}$ do perímetro urbano (Figura). A vegetação era constituída, principalmente, de árvores frutíferas e gramíneas, com algumas árvores nativas e outras replantadas. O sítio Flor de Maio apresenta as seguintes características: 1) há quatro casas muito próximas uma das outras, rodeadas por vários tipos de árvores frutíferas, principalmente jabuticabeiras e mangueiras; 2) dentre os animais, destacam-se cães e galinhas, que circulam livremente pelo terreno em volta das casas, e os animais de maior porte (bovinos e eqüinos) ocupam um pasto. Há também suínos, que permanecem confinados em chiqueiro; 3) nos fundos do sítio passam dois pequenos córregos (sem nome) com uma pequena mata ciliar e vazão de água apenas nos períodos de chuvas intensas; 4) os abrigos de animais domésticos e as residências estão próximos uns dos outros; 5) existe acúmulo de matéria orgânica (folhas e frutos caídos), resíduos agrícolas, fezes de animais domésticos e restos de alimentos a eles oferecidos, bem como grande umidade do solo provocada pelo descarte de águas de uso cotidiano e pelo sombreado das árvores.

As coletas de flebotomíneos foram realizadas durante 60 dias de 26 de janeiro a 28 de fevereiro e de 23 de março a 22 de abril de 2004 , no peridomicílio, das $20 \mathrm{~h}$ às $24 \mathrm{~h}$ num total de $120 \mathrm{~h}$ de coleta por armadilha, em cada um dos dois períodos. O horário de coleta foi escolhido tendo em vista que no norte do Paraná a frequiência desses insetos tem sido elevada, especialmente das $21 \mathrm{~h}$ às $6 \mathrm{~h} .{ }^{12}$ Nas coletas foram utilizadas quatro armadilhas luminosas tipo Falcão (AF), instaladas simultaneamente, ao lado de quatro gaiolas destinadas a abrigar os animais utilizados como iscas. As AF foram numeradas, distribuídas e instaladas ao lado de quatro gaiolas, contendo os animais, numa área de $25 \mathrm{~m}^{2}$, localizada no pomar. Cada uma das AF ocupou o vértice de um dos ângulos da área.

O rodízio dos animais foi feito a partir da AF1, diariamente, no sentido horário, seguindo um cronograma previamente determinado.

Como o número de flebotomíneos no primeiro período de coletas foi muito inferior ao de uma coleta feita em abril de 2003 por agentes da Fundação Nacional de Saúde (Funasa) na mesma localidade, decidiu-se pela inclusão de mais um período de coletas para melhorar a confiabilidade da análise estatística.
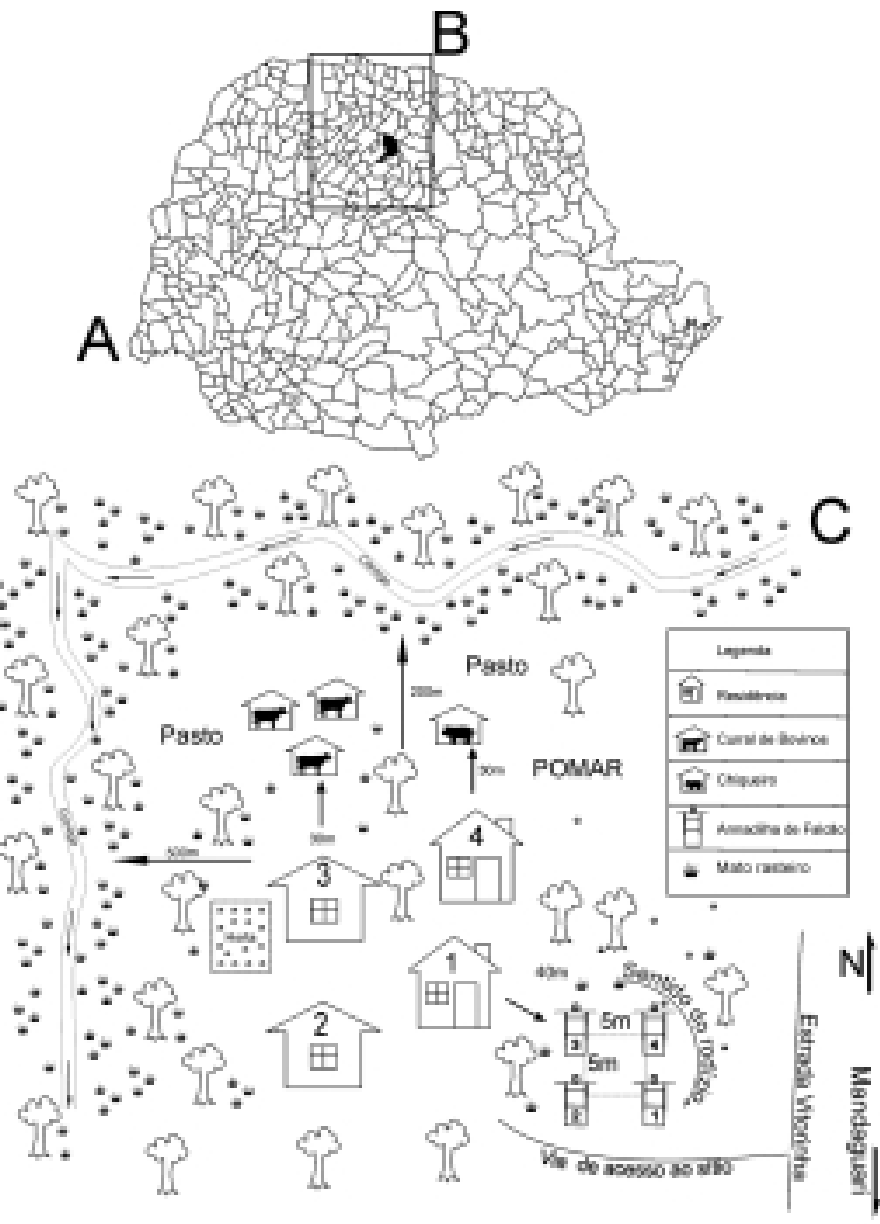

A: Estado do Paraná

B: Município de Mandaguari

o Flor de Maio

igura - Diagrama do local de pesquisa mostrando os pontos de instalação das armadilhas de Falcão no Sítio Flor de Maio. Mandaguari, PR, 2004.

Os animais escolhidos para a realização do estudo foram: um cão (Canis familiaris, Linnaeus), uma leitoa (Sus scrofa domesticus, Linnaeus), três galinhas (Gallus domesticus, Linnaeus) e dois coelhos (Oryctolagus cuniculus, Linnaeus). Este procedimento foi uma tentativa de oferecer aos flebotomíneos iscas com pesos mais ou menos equivalentes; galinhas e coelhos, com menor porte, foram usados em maior número, para evitar uma atração maior dos insetos pelo suíno ou cão, de maior porte. Os animais foram escolhidos pelo fato de serem domésticos e de fácil manejo, viverem no peridomicílio, possuírem pequeno porte e/ou serem potenciais reservatórios de Leishmania.

As gaiolas usadas para abrigar os animais durante as coletas foram confeccionadas em madeira, com $0,70 \mathrm{~m}$ de altura e área de $0,415 \mathrm{~m}^{2}(0,50 \mathrm{~m} \mathrm{X} \mathrm{0,83}$ $\mathrm{m})$. Entre um balaústre e outro das grades que constituíam as gaiolas foi deixado um espaço de $5 \mathrm{~cm}$. Entre uma coleta e outra as gaiolas foram lavadas 
Tabela 1 - Número de flebotomíneos coletados em dois períodos segundo espécie e sexo, sítio Flor de Maio. Município de Mandaguari, PR, 2004.

\begin{tabular}{lcccccccc}
\hline Mês/Ano & \multicolumn{3}{c}{$26 / 1$ a $28 / 2$ de 2004} & \multicolumn{3}{c}{$23 / 3$ a $22 / 2$ de 2004} \\
Espécies & M & F & Total & $\%$ & M & F & Total & $\%$ \\
\hline Nyssomyia whitmani & 236 & 521 & 757 & 78,1 & 149 & 360 & 509 & 69,9 \\
Pintomyia fischeri & 125 & 52 & 177 & 18,3 & 110 & 46 & 156 & 21,5 \\
Migonemyia migonei & 16 & 5 & 21 & 2,2 & 33 & 12 & 45 & 6,2 \\
Nyssomyia neivai & 5 & 3 & 8 & 0,8 & 12 & 4 & 16 & 2,2 \\
Pintomyia pessoai & 1 & 2 & 3 & 0,3 & 0 & 1 & 1 & 0,1 \\
Psathiromyia shannoni & 2 & 1 & 3 & 0,3 & 1 & 0 & 1 & 0,1 \\
\hline Total & 385 & 584 & 969 & 100 & 305 & 423 & 728 & 100 \\
\hline
\end{tabular}

M: Macho; F: Fêmea

com água para eliminar os resíduos orgânicos deixados pelos animais.

Durante o período de captura, procurou-se não interferir no ambiente e não houve quaisquer alterações de relevância praticadas pelos moradores que pudessem interferir nos resultados.

Os flebotomíneos capturados foram sacrificados com clorofórmio, armazenados em caixas apropriadas de papelão impregnadas com naftalina, e encaminhados ao laboratório para identificação. A clarificação desses insetos foi feita em solução de hidróxido de potássio $(\mathrm{KOH})$ a $20 \%, 70^{\circ} \mathrm{C}$, durante $90 \mathrm{~min}$. Em seguida, os flebotomíneos foram identificados em microscópio binocular com aumento de 100 ou 200 vezes.

A nomenclatura das espécies de flebotomíneos segue Galati (2003). ${ }^{7}$

A análise quantitativa de flebotomíneos coletados foi feita por testes estatísticos não paramétricos, utilizando o programa Statistica 6.1.Utilizou-se o nível de significância a 5\%, como critério para estabelecer a validade estatística para as variações encontradas.

Para analisar as quantidades de flebotomíneos capturados em relação aos animais, e as armadilhas e espécies em cada período (amostras independentes), foi usado ANOVA por ranks via Kruskall-Wallis, complementada pelo teste de comparações múltiplas. Esta análise abrangeu apenas as fêmeas de flebotomíneos, uma vez que somente estas são hematófagas.

Os dados de temperatura e umidade relativa do ar foram coletados diariamente. Os dados relativos à precipitação pluviométrica foram gentilmente cedidos pela Cooperativa dos Cafeicultores de Mandaguari (COCARI).

\section{RESU LTAD O $S$}

Observa-se na Tabela 1 que foram capturados 969 exemplares de flebotomíneos, de janeiro a fevereiro, e 728 de março a abril de 2004, num total de 1.697 exempla- res, sendo 59,3\% fêmeas. As espécies coletadas foram: Nyssomyia whitmani (Antunes \& Coutinho), Pintomyia fischeri (Pinto), Migonemyia migonei (França), Nyssomyia neivai (Pinto), Pintomyia pessoai (Coutinho \& Barretto), Psathyromyia shannoni (Dyar), considerando os dois períodos de coletas. A espécie predominante foi $N$. whitmani, em ambos os períodos de coletas, com $78,1 \%$ e $69,9 \%$ dos exemplares capturados, respectivamente.

A Tabela 2 mostra que no primeiro período de coletas as médias diárias de fêmeas de $N$. whitmani, coletadas em cada armadilha, foram estatisticamente diferentes $(\mathrm{p}=0,0005)$, sendo a menor média $(1,19)$ obtida na $\mathrm{AF} 1$. No segundo período, as médias diárias coletadas em cada AF foram estatisticamente diferentes $(\mathrm{p}=0,0194)$, sendo que a média diária da AF3 $(4,83)$ foi significativamente diferente da AF1 e AF4 $(1,90)$; porém, não diferiu da $\mathrm{AF} 2(3,37)$. A mesma tabela mostra que não houve diferença estatisticamente significante entre as quantidades de fêmeas de $P$. fischeri capturados nas AF, no primeiro ( $\mathrm{p}=0,061)$ e no segundo período $(\mathrm{p}=0,5078)$. As armadilhas com letras ( $a$, $\mathrm{b}, \mathrm{c})$ iguais representam média de ranks iguais.

A Tabela 3 mostra que não houve diferença significativa entre as médias diárias de fêmeas de $N$. whitmani, em relação aos animais utilizados como iscas no primeiro período de coleta $(\mathrm{p}=0,1470)$, nem no segundo $(\mathrm{p}=0,6232)$. Esta Tabela mostra que nos dois períodos de coletas não houve diferenças significativas nas médias diárias das fêmeas de $P$. fischeri, entre os animais utilizados como iscas no estudo. As armadilhas com letras $(a, b, c)$ iguais representam média de ranks iguais.

\section{DISCUSSÃO}

As espécies capturadas N. whitmani, N. neivai, P. fischeri, P. pessoai, M. migonei e $P$. shannoni foram constatadas anteriormente em municípios das mesorregiões norte-central ${ }^{9,10,13}$ e noroeste do Estado do Paraná., ${ }^{42-14}$

No sítio Flor de Maio, numa única noite de abril de 
Tabela 2 - Número de fêmeas de Nyssomyia whitmani e Pintomyia fischeri coletadas em armadilhas de Falcão, em dois períodos, no sítio Flor de Maio. Município de Mandaguari, PR, 2004.

\begin{tabular}{|c|c|c|c|}
\hline Armadilha & $\mathrm{N}$ & Média diária & Média de ranks \\
\hline $\begin{array}{l}\text { AF1 } \\
\text { AF2 } \\
\text { AF3 } \\
\text { AF4 } \\
\text { Total }\end{array}$ & $\begin{array}{c}26 \text { de } \\
38 \\
161 \\
174 \\
148 \\
521 \\
23\end{array}$ & $\begin{array}{r}\text { de } 2004 * \\
1,19 \\
5,03 \\
5,44 \\
4,63 \\
16,28 \\
2004^{* *}\end{array}$ & $\begin{array}{c}41,4 \\
73,8 \\
75,17 \\
67,63\end{array}$ \\
\hline $\begin{array}{c}A F 1^{\mathrm{a}} \\
A F 2^{\mathrm{abc}} \\
A F 3^{\mathrm{c}} \\
\text { AF4 }^{\mathrm{ab}} \\
\text { Total }\end{array}$ & $\begin{array}{c}57 \\
101 \\
145 \\
57 \\
360\end{array}$ & $\begin{array}{r}1,90 \\
3,37 \\
4,83 \\
1,90 \\
12,00\end{array}$ & $\begin{array}{l}49,48 \\
66,30 \\
73,50 \\
52,72\end{array}$ \\
\hline
\end{tabular}

Pintomyia fischeri

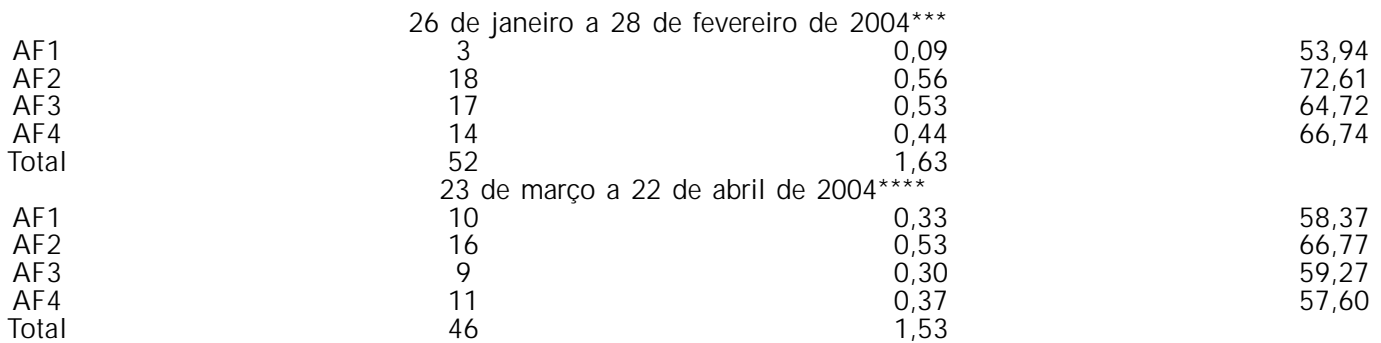

Armadilhas com letras iguais representam média de ranks iguais.

${ }^{*} p=0,0005$; em duas noites deste período as coletas não foram realizadas; ${ }^{*} p<0,0194$ (Anova de Kruskal-Wallis); ${ }^{* * *} p=0,061 ;$ $* * * * p=0,5078$ (Anova de Kruskal-W allis)

2003, em quatro horas, agentes de saúde da Funasa* capturaram 3.057 exemplares de flebotomíneos. A diferença da quantidade de flebotomíneos capturados em abril de 2003 e a do presente trabalho pode ser justificada sob os seguintes aspectos: 1) após aquela coleta foi feita desinsetização das casas e dos abrigos de animais com piretróides; $*$ 2) entre aquela coleta e as do presente trabalho foi feito um plantio de feijão, que exigiu o uso de inseticidas, para o controle do "manhoso" (Chacodermus bimaculatus) e da mosca branca, respectivamente;** 3) durante o ano de 2003, a precipitação pluviométrica foi de $1.519 \mathrm{~mm}$, abaixo, portanto, da média anual de $1.742 \mathrm{~mm}$. De dezembro de 2002 a abril de 2003 as médias diárias de precipitação foram mais intensas e mais bem distribuídas do que as de dezembro de 2003 a abril de 2004, ${ }^{* * *}$ o que manteve o solo com maior teor de umidade na coleta de flebotomíneos de abril de 2003 no sítio Flor de Maio.

A espécie $N$. whitmani tem também predominado em toda mesorregião Norte Central Paranaense., ${ }^{9,10,12,14}$ A prevalência de $N$. whitmani nos municípios dessa mesorregião parece, dentre outros fatores, estar relacionada às áreas de latossolos vermelhos e vermelhoamarelos ou nitossolos, que retêm mais umidade.

A segunda espécie mais prevalente foi $P$. fischeri; o que já foi constatado nos municípios de Londrina,
Apucarana, Cambira, Marumbi, Arapongas, Sabáudia e Mandaguari (sítio Flor de Maio), todos localizados na mesorregião Norte Central Paranaense. ${ }^{10, *}$

Embora o número de exemplares de M. migonei tenha sido irrelevante do ponto de vista estatístico, sua importância na epidemiologia da LTA não pode ser desconsiderada, uma vez que no Estado de São Paulo esse inseto foi associado à transmissão de LTA. ${ }^{2}$

Constatou-se que nos dois períodos de coletas a AF1, independentemente da isca animal utilizada, teve menor rendimento. Esse fato pode estar relacionado à posição na qual a armadilha se encontrava, ao lado de uma via de acesso às residências. Além disso, a vegetação próxima à armadilha era mais escassa, tornando o ambiente ao redor mais aberto em relação às posições das demais armadilhas.

Entre os animais utilizados como iscas, o coelho foi o que menos atraiu fêmeas de $N$. whitmani no primeiro período de coletas. Este fato pode ter ocorrido em função de este ter sido o único animal distinto da fauna de animais domésticos mais comuns na zona rural. No entanto, como esta diferença não foi observada no segundo período de coletas, pode-se inferir que as fêmeas de $N$. whitmani ajustaram o seu padrão alimentar ao coelho. Pela análise do conteúdo intestinal de fêmeas 
Tabena 3 - Número de fêmeas de Nyssomyia whitmani e Pintomyia fischeri coletadas em armadilhas de Falcão com iscas, em dois períodos, no sítio Flor de Maio. Município de Mandaguari, PR, 2004.

\begin{tabular}{|c|c|c|c|}
\hline \multicolumn{4}{|c|}{ Nyssomyia whitmani } \\
\hline $\begin{array}{c}\text { Porco }^{\mathrm{a}} \\
\text { Cachorro } \\
\text { Galinhab }^{\mathrm{ab}} \\
\text { Coelho }^{\mathrm{b}} \\
\text { Total }\end{array}$ & $\begin{array}{l}\text { e jane } \\
165 \\
109 \\
165 \\
82 \\
521 \\
\text { de } \mathrm{m}\end{array}$ & $\begin{array}{r}2004 * \\
5,16 \\
3,41 \\
5,16 \\
2,56 \\
16,28 \\
2004 * *\end{array}$ & $\begin{array}{l}70,67 \\
66,94 \\
68,64 \\
51,75\end{array}$ \\
\hline $\begin{array}{c}\text { Porco }^{\mathrm{a}} \\
\text { Cachorro }^{\mathrm{ab}} \\
\text { Galinha }^{\mathrm{ab}} \\
\text { Coelho } \\
\text { Total }\end{array}$ & $\begin{array}{c}89 \\
64 \\
94 \\
113 \\
360\end{array}$ & $\begin{array}{c}2,97 \\
2,13 \\
3,13 \\
3,77 \\
12,00\end{array}$ & $\begin{array}{l}62,72 \\
54,65 \\
58,95 \\
65,68\end{array}$ \\
\hline \multicolumn{4}{|c|}{ Pintomyia fischeri } \\
\hline $\begin{array}{c}\text { Porco }^{\mathrm{a}} \\
\text { Cachorro }^{\mathrm{ab}} \\
\text { Galinha }^{\mathrm{ab}} \\
\text { Coelho } \\
\text { Total }\end{array}$ & $\begin{array}{l}\text { jane } \\
13 \\
17 \\
11 \\
11 \\
52 \\
\text { de ma }\end{array}$ & $\begin{array}{r}2004 * * * \\
0,41 \\
0,53 \\
0,34 \\
0,34 \\
1,62 \\
04 * * * *\end{array}$ & $\begin{array}{l}64,52 \\
72,17 \\
65,70 \\
55,61\end{array}$ \\
\hline $\begin{array}{c}\text { Porco }^{\mathrm{a}} \\
\text { Cachorro }^{\mathrm{ab}} \\
\text { Galinha }^{\mathrm{ab}} \\
\text { Coelho } \\
\text { Total }\end{array}$ & $\begin{array}{c}10 \\
6 \\
21 \\
9 \\
46\end{array}$ & $\begin{array}{l}0,33 \\
0,20 \\
0,70 \\
0,30 \\
1,53 \\
\end{array}$ & $\begin{array}{l}59,73 \\
56,50 \\
66,50 \\
59,27\end{array}$ \\
\hline
\end{tabular}

Armadilhas com letras iguais representam média de ranks iquais.

${ }^{*} p=0,1470 ;{ }^{* *} p=0,6232$ (Anova de Kruskal-W allis); ${ }^{* * *} p=0,1305 ; * * * p=0,5048$ (Anova de Kruskal-Wallis)

de flebotomíneos, pelo teste de precipitina, CamargoNeves et al, ${ }^{3}$ Morrison et al, ${ }^{11}$ Christensen et $\mathrm{al}^{5}$ e Tesh et $\mathrm{a}^{17}$ notaram que certas espécies desses dípteros mostravam maior afinidade alimentar por mamíferos (suíno, bovino, cão, eqüino, homem ou roedores) ou aves. Contudo, Casanova* verificou que esses insetos ajustam seu padrão alimentar à disponibilidade de hospedeiros. Christensen et $\mathrm{al}^{5}$ ressalvaram que, apesar da afinidade desses insetos por determinados hospedeiros, eles eram oportunistas, pois se alimentavam do sangue de diversos tipos de mamíferos. Tesh et a ${ }^{17}$ observaram, ainda, que esses insetos tiveram maior afinidade por roedores, mas mostraram também maior amplitude de hospedeiros. Tesh et $\mathrm{al}^{16}$ verificaram que a variação do hábito alimentar desses dípteros, provavelmente, refletia diferenças na composição da fauna das localidades de coletas. Bongiorno et al, ${ }^{1}$ pelo método dot-ELISA, concluíram que a afinidade desses insetos parece ter maior relação com a disponibilidade do animal hospedeiro (número, tamanho) do que a uma atração específica.

Os resultados mostraram que as espécies $N$. whitmani e P. fischeri são oportunistas e, provavelmente, suas fêmeas ajustam os seus hábitos alimentares à disponibilidade de hospedeiros. Assim, a flexibilidade alimentar de determinadas espécies de flebotomíneos, conforme a disponibilidade de fontes sangüíneas, nos ambientes antrópicos, sugerem o ecletismo alimentar desses insetos. As técnicas laboratoriais (reação de precipitina, ELISA, Western-blot e outras) permitem esclarecer melhor o hábito alimentar de flebotomíneos. Contudo, o presente estudo contribui para o conhecimento dos hábitos alimentares das espécies de flebotomíneos locais.

\section{AG RADECIMENTOS}

À Cooperativa de Cafeicultores de Mandaguari (COCARI), Empresa Paranaense de Assistência Técnica e Extensão Rural (EMATER/Mandaguari) e Prefeitura do Município de Mandaguari pelas informações sobre a meteorologia, ocupação do solo, tipo de solo e características do terreno; ao sr. Edimilson Carlos Altomani, do sítio Flor de Maio, pelo apoio na coleta de flebotomíneos. 


\section{REFERÊNCIAS}

1. Bongiorno G, Habluetzel A, Khoury C, Maroli M. Host preferences of phlebotomine sand flies at a hypoendendemic focus of canine leishmaniasis in central Italy. Acta Tropica. 2003;88:109-16.

2. Camargo-Neves VLF, Gomes AC, Antunes JL. Correlação da presença de espécies de flebotomíneos (Diptera: Psychodidae) com registros de casos da leishmaniose tegumentar americana no estado de São Paulo, Brasil. Rev Soc Bras Med Trop. 2002;35:299-306.

3. Camargo-N eves VLF, Rodas LAC, Poletto DW, Gomes AC. Feeding habit of Lutzomyia longipalpis in Araçatuba county, state of São Paulo, Brazil. Entomol Vectores. 2002;9(Supl 1):63.

4. Campbell-Lendrum DH, Brandão-Filho SP, Pinto MC, Vexenat A, Davies CR. Domesticity of Lutzomyia whitmani (Diptera: Psychodidae) populations: field experiments indicate bahavioural differences. Bull Entomol Res. 2000;90:41-8.

5. Christensen HA, Herrer A. Panamian Lutzomyia (Diptera: Psychodidae) host attraction profiles. J Med Entomol. 1980;17:522-8.

6. Dias FOP, Lorosa ES, Rebelo JMM. Fonte alimentar sangüínea e a peridomiciliação de Lutzomyia longipalpis (Lutz \& Neiva, 1912) (Psychodidae, Phlebotominae). Cad Saúde Pública. 2003;19:1373-80.

7. Galati EAB. Flebotomíneos do Brasil. In: Rangel EF, Lainson R. Morfologia e taxonomia. Rio de Janeiro: Fiocruz; 2003. p. 23-51.

8. Lima AP, Minelli L, Comunello E, Teodoro U. Distribuição da leishmaniose tegumentar por imagens de sensoriamento remoto orbital, no estado do Paraná, sul do Brasil. An Bras Dermatol. 2002;77:681-92.

9. Luz E, Membrive N, Castro EA, Dereure J, Pratlong J, Dedet $A$, et al. Lutzomyia whitmani (Diptera: Psychodidae) as vector of Leishmania (V.) braziliensis in Paraná state, southern Brazil. Ann Trop Med Parasitol. 2000;94:623-31.
10. Membrive NA, Rodrigues $G$, Membrive $U$, Monteiro WM, Neitzke HC, Lonardoni MVL, et al.

Flebotomíneos de municípios do norte do estado do Paraná, sul do Brasil. Entomol Vectores. 2004;11:673-80.

11. Morrison AC, Ferro C, Tesh RB. Host preferences of sand fly $L$. longipalpis at an endemic focus of amercian visceral leishmaniasis in Colombia. Am J trop Med Hyg. 1993;49:68-75.

12. Teodoro U. Características ecológicas de Flebotomíneos (Diptera Psychodidae) em habitats antrópicos, município de Jussara, Paraná, Brasil. Rev Soc Bras Med Trop. 1996;29:625-6.

13. Teodoro U, Silveira TGV, Santos DR, Santos ES, Santos AR, Oliveira $O$, et al. Freqüência da fauna de flebotomíneos no domicílio e em abrigos de animais domésticos no peridomicílio, nos municípios de Cianorte e Doutor Camargo - estado do Paraná Brasil. Rev Patol Trop. 2001;30:209-33.

14. Teodoro U, Kühl JB, Abbas M, Dias AC. Luz e aves como atrativos de flebotomíneos (Diptera, Psychodidae), no sul do Brasil. Rev Bras Entomol. 2001;45:167-72.

15. Teodoro U, Silveira TGV, Santos DR, Santos ES, Santos AR, Oliveira $O$, et al. Influência da reorganização, da limpeza do peridomicílio e da desinsetização de edificações na densidade populacional de flebotomíneos, no município de Doutor Camargo, estado do Paraná, Brasil. Cad Saúde Pública. 2003;19:1801-13.

16. Tesh RB, Chaniotis BN, Aronson MD, Johnson KM. $N$ atural host preferences of Panamanian phlebotomine sandflies as determined by precipitin test. Am J Trop Med Hyg. 1971;20:150-6.

17. Tesh RB, Chaniotis BN, Carrera BR, Johnson KM. Further studies on natural preferences of Panamanian phlebotomine sandflies. Am J Epidemiol. 1972;95:88-93. 